\title{
INDUCTIVE LIMITS OF VECTOR-VALUED SEQUENCE SPACES
}

\author{
JosÉ BONET, SUSANNE DIEROLF AND CARMEN FERNÁNDEZ
}

Abstract

Let $L$ be a normal Banach sequence space such that every element in $L$ is the limit of its sections and let $E=$ ind $E_{n}$ be a separated inductive limit of locally convex spaces. Then ind $L\left(E_{n}\right)$ is a topological subspace of $L(E)$.

The aim of this note is to prove the following result on the interchangeability of inductive limits and spaces of vector valued sequences: if $L$ is a normal Banach sequence space with the property that every element of $L$ is the limit of its sections and $E=$ ind $E_{n}$ is a separated locally convex inductive limit, then the inductive linit ind $L\left(E_{n}\right)$ is a topological subspace of $L(E)$. The situation is completely different for the sequence space $L=1^{\infty}$. In fact the first two authors showed in [2] that there are even strict inductive limits of Fréchet spaces $E=$ ind $E_{n}$ such that the canonical injection ind $1^{\infty}\left(E_{n}\right) \subset 1^{\infty}(E)$ is not open.

In what follows $(L,\|\|)$ denotes a normal Banach sequence space, i.e., a Banach space that satisfies

( $\alpha) \varphi \subset L \subset \omega$ algebraically and the inclusion $(L,\|\|) \subset \omega$ is continous.

( $\beta) \forall a=\left(a_{k}\right)_{k \in N} \in L \forall b=\left(b_{k}\right)_{k \in N} \in \omega$ such that $\left|b_{k}\right| \leq\left|a_{k}\right| \forall k \in \mathbf{N}$, we have that $b \in L$ and $\|b\| \leq\|a\|$.

We will also assume the following property (cf $\{1\})$

(c) $\lim _{n \rightarrow \infty}\left\|\left((0)_{k<n},\left(a_{k}\right)_{k \geq n}\right)\right\|=0, \forall a=\left(a_{k}\right)_{k \in N} \in L$.

This property is sometimes called AK-property. Clearly $(L,\|\|)=1^{\infty}$ does not satisfy $(\varepsilon)$, whereas $(L,\|\|)=1^{p}, 1 \leq p$ o or $c_{0}$ has property $(\varepsilon)$.

We observe that there is $\left(\mu_{k}\right)_{k \in N} \in L$ with $\mu_{k}>0(k \in N)$ and $\left\|\left(\mu_{k}\right)_{k \in N}\right\|=1$

Given a locally convex space $E$, we denote by $\operatorname{cs}(E)$ the family of all continuous seminorms on $E$. Given $E$ the vector valued sequence space $L(E)$ is defined by

$$
L(E)=\left\{x=\left(x_{k}\right)_{k \in \mathbb{N}} \in E^{N} ;\left(r\left(x_{k}\right)_{k \in N}\right) \in L \text { for all } r \in c s(E)\right\}
$$


endowed with the locally convex topology defined by the seminorms

$$
x \longrightarrow\left\|\left(r\left(x_{k}\right)_{k \in \mathbb{N}}\right)\right\|
$$

as $r$ varies in $c s(E)$. Clearly if $(L,\|\|)$ satisfies property $(\varepsilon)$, then the countable direct sum $\oplus\{E: n \in \mathbb{N}\}=E^{(\mathbb{N})}$ is dense in $L(E)$.

Given a separated locally convex inductive limit $E=$ ind $E_{n}$ we are interested in the following question: is ind $L\left(E_{n}\right)$ a topological subspace of $L(E)$ ?. If $(L,\|\|)=1^{1}$, a positive answer follows from a classical result of Grothendieck on projective tensor products (see e.g. [4]). If $(L,\|\|)=c_{0}$ the positive answer is a particular case of a result of Mujica $[5, I, 7]$. We prove now that the answer is positive for arbitrary $(L,\|\|)$ satisfying $(\varepsilon)$.

1. Proposition. Let $E$ be a locally convex space, $F$ a closed subspace of $E$ and $q: E \longrightarrow E / F$ the canonical surjection. The mapping $Q: L(E) \longrightarrow$ $L(E / F)$ defined by $Q\left(\left(x_{k}\right)_{k \in \mathbb{N}}\right):=\left(q\left(x_{k}\right)\right)_{k \in \mathbb{N}}$ is open onto its image. If $E$ is a Fréchet space then $Q$ is also surjective.

Proof: Since $E^{(\mathbf{N})}$ is a dense subspace of $L(E)$ and $Q\left(E^{(\mathbf{N})}\right)=(E / F)^{(\mathbb{N})}$, according to $[4,32,5(3)]$ it is enough to show that $Q: E^{(\mathbb{N})} \longrightarrow(E / F)^{(\mathbb{N})}$ is open. To do this we fix $r \in c s(E)$ and we show

$$
\begin{aligned}
& Q\left(\left\{x \in L(E) ; x \in E^{(\mathbb{N})}\left\|\left(r\left(x_{k}\right)\right)_{k \in \mathbb{N}}\right\| \leq 1\right\}\right) \supset \\
& \left\{\tilde{x} \in L(E / F) ; \tilde{x} \in(E / F)^{(\mathbb{N})}\left\|\left(\tilde{r}\left(\tilde{x}_{k}\right)\right)_{k \in \mathbb{N}}\right\| \leq 2^{-1}\right\}
\end{aligned}
$$

where $\tilde{r}(z+F):=\inf \{r(z+y) ; y \in F\}(z \in E)$ is the quotient seminorm. We fix $\left.\left(\mu_{k}\right)_{k \in \mathbb{N}} \in L, \mu_{k}>0(k \in \mathbb{N}), \|\left(\mu_{k}\right)_{k \in \mathbb{N}}\right) \|=1$. Given $\tilde{x} \in(E / F)^{(\mathbb{N})}$ with $\left\|\left(\tilde{r}\left(\tilde{x}_{k}\right)\right)_{k \in \mathbb{N} \|}\right\| \leq 2^{-1}$ we find $1 \in \mathbb{N}$ such that $\tilde{x}_{k}=0$ for $1 \leq k$. For each $k<1$ we select $y \in F$ such that $r\left(x_{k}+y_{k}\right)<\tilde{r}\left(x_{k}+F\right)+2^{-1} \mu_{k}$. Then $\left.x=\left(\left(x_{k}+y_{k}\right)_{k<1},(0)_{1 \leq k}\right)\right)$ belongs to $E^{(N)} \subset L(E), Q(x)=\tilde{x}$ and $\left.\|\left(\left(r\left(x_{k}+y_{k}\right)\right)_{k<1},(0)_{1 \leq k}\right)\right) \| \leq 1$.

If $E$ is also a Fréchet space, then $Q(L(E))$ is a Fréchet space dense in $L(E / F)$. Consequently $Q$ is surjective.

2. Proposition. Let $\left(E_{n}\right)_{n \in \mathbb{N}}$ be a sequence of locally convex spaces. Then the map $\psi: L\left(\oplus\left\{E_{n}: n \in \mathbb{N}\right\}\right) \longrightarrow \oplus\left\{L\left(E_{n}\right): n \in \mathbb{N}\right\}$ defined by

$$
\psi\left(\left(\left(x_{n}^{k}\right)_{n \in \mathbb{N}}\right)_{k \in \mathbb{N}}\right):=\left(\left(x_{n}^{k}\right)_{k \in \mathbb{N}}\right)_{n \in \mathbb{N}}
$$

is a topological isomorphism.

Proof: Given $x=\left(\left(x_{n}^{k}\right)_{n \in \mathbb{N}}\right)_{k \in \mathbb{N}}$ in $L\left(\oplus\left\{E_{n}: n \in \mathbb{N}\right\}\right)$, to show that $\psi(x) \in$ $\oplus\left\{L\left(E_{n}\right): n \in \mathbf{N}\right\}$ it is enough to see that there is $m \in \mathbb{N}$ such that $x_{n}^{k}=0$ for all $n \geq m, k \in \mathbf{N}$. If we assume the contrary we can find two strictly increasing 
sequences $(k(j))_{j \in N}$ and $(n(j))_{j \in N}$ such that $x_{n(j)}^{k(j)} \neq 0$ for all $j \in N$ (recall that each $\left(x_{n}^{k}\right)_{n \in N}$ belongs to $\left.\oplus\left\{E_{n}: n \in N\right\}\right)$. We select $\left(\lambda_{k}\right)_{k \in N} \in \omega \backslash L$ with $\lambda_{k(j)}>0$ for all $j \in N$ and $\lambda_{k}=0$ if $k \notin\{k(j) ; j \in N\}$. For all $j \in N$ we find $r_{j} \in \operatorname{cs}\left(E_{n(j)}\right)$ with $r_{j}\left(x_{n(j)}^{k(j)}\right)$ greater than $\lambda_{k(j)}$. It is clear that $r\left(\left(z_{n}\right)_{n \in N}\right)=$ $=\sum_{j=1}^{\infty} r_{j}\left(z_{n(j)}\right)$ defines a continous seminorm on $\oplus\left\{E_{n}: n \in N\right\}$. Therefore for $x^{k}:=\left(x_{n}^{k}\right)_{n \in \mathbb{N}}(k \in \mathbb{N})$, we have $\left.\left(r\left(x^{k}\right)\right)\right) \in L$. But $r\left(x^{k(j)}\right) \geq r_{j}\left(x_{n(j)}^{k(j)}\right)>\lambda_{k(j)}$, for all $j \in \mathbf{N}$ and $0=\lambda_{k} \leq r\left(x^{k}\right)$ if $k \notin\{k(j) ; j \in \mathbb{N}\}$. Consequently $\left(\lambda_{k}\right)_{k \in N} \in$ $L$, a contradiction. Therefore $\psi$ is well defined. Clearly $\psi$ is linear and injective. To show that $\psi$ is surjective, we take $x=\left(\left(x_{n}^{k}\right)_{k \in \mathbb{N}}\right)_{n \in \mathbb{N}}$ in $\oplus\left\{L\left(E_{n}\right): n \in \mathbb{N}\right\}$. Clearly $\left(x_{n}^{k}\right)_{n \in \mathbb{N}} \in \oplus\left\{E_{n} ; n \in \mathbb{N}\right\}$ for all $k \in \mathbb{N}$, since $x_{n}^{k}=0$ for all $n \geq m$ and $k \in \mathbb{N}$. Given $r \in \operatorname{cs}\left(\oplus\left\{E_{n} ; n \in \mathbb{N}\right\}\right)$ we can find $r_{n} \in \operatorname{cs}\left(E_{n}\right) n \in \mathbf{N}$, with $r(z) \leq \max \left(r_{n}\left(z_{n}\right) ; n \in \mathbb{N}\right)$ for all $z=\left(z_{n}\right) \in \oplus\left\{E_{n} ; n \in \mathbb{N}\right\}$. Therefore for all $k \in \mathbb{N}$

$$
r\left(\left(x_{n}^{k}\right)_{n \in N}\right) \leq \max \left(r_{n}\left(x_{n}^{k}\right) ; 1 \leq n \leq m\right) \leq \sum_{n=1}^{m} r_{n}\left(x_{n}^{k}\right)
$$

Since $\left(r_{n}\left(x_{n}^{k}\right)_{k \in \mathbb{N}}\right) \in L$ for $1 \leq n \leq m$, we conclude $y=\left(\left(x_{n}^{k}\right)_{n \in \mathbb{N}}\right)_{k \in \mathbb{N}} \in$ $L\left(\oplus\left\{E_{n} ; n \in \mathbb{N}\right\}\right)$ and $\psi(y)=x$.

Now the continuity of $\psi^{-1}: \oplus\left\{L\left(E_{n}\right) ; n \in \mathrm{N}\right\} \longrightarrow L\left(\oplus\left\{E_{n} ; n \in \mathrm{N}\right\}\right)$ follows from the fact that its restriction to each $L\left(E_{n}\right)$ is clearly continuous. Finally we show that $\psi$ is continuous. To do this we consider $r_{n} \in c s\left(E_{n}\right)(n \in N)$ and we observe that

$$
\left.\sup _{n \in \mathbb{N}} \|\left(r_{n}\left(x_{n}^{k}\right)\right)_{k \in \mathbb{N}}\right)\|\leq\|\left(\sup _{n \in \mathbb{N}}\left(r_{n}\left(x_{n}^{k}\right)\right)_{k \in \mathbb{N}}\right) \|
$$

holds for every $\left(\left(x_{n}^{k}\right)_{n \in \mathbf{N}}\right)_{k \in \mathbb{N}} \in L\left(\oplus\left\{E_{n} ; n \in \mathbf{N}\right\}\right)$.

3. Theorem. Let $(L,\|\|$.$) be a normal Banach sequence space with property$ (c). Let $E=$ ind $E_{n}$ be a separated locally convex inductive limit. Then find $L\left(E_{n}\right)$ is a topological subspace of $L\left(\right.$ ind $\left.E_{n}\right)$.

Proof: We consider the following diagram

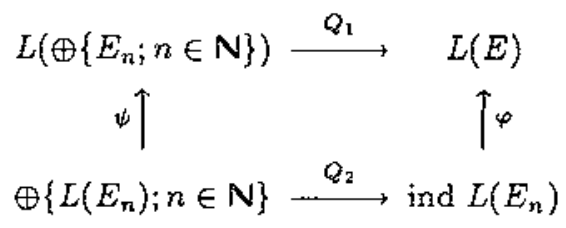

where, for $q_{1}: \oplus\left\{E_{n} ; n \in \mathbb{N}\right\} \longrightarrow E$ the canonical quotient map $q_{1}\left(\left(z_{n}\right)_{n \in \mathbb{N}}\right)=$ $=\sum_{n=1}^{\infty} z_{n}$ we define $Q_{1}\left(\left(x_{k}\right)_{k \in N}\right)=\left(q_{1}\left(x_{k}\right)\right)_{k \in \mathbb{N}}$ for all $\left(x_{k}\right)_{k \in \mathbb{N}}$ in 
$L\left(\oplus\left\{E_{n} ; n \in \mathbb{N}\right\}\right) . Q_{2}$ is the canonical quotient $\operatorname{map}$ and $\varphi$ is the canonical injection which is continous. According to proposition $1, Q_{1}$ is open onto its image. Certainly $Q_{2}$ is open and $\psi^{-1}$ is a topological isonorphism, according to proposition 2. Since the diagram is commutative, it follows that $\varphi$ is also open onto its image. Thus ind $L\left(E_{n}\right)$ is a topological subspace of $L(E)$.

4. Corollary. Let $(L,\|\|)$ be a normal Banach sequence space with property $(\varepsilon)$. Let $E=$ ind $E_{n}$ be a strict inductive limit of locally convex spaces with $E_{n}$ closed in $E_{n+1}$ for all $n \in \mathbb{N}$. Then $L(E)=$ ind $L\left(E_{n}\right)$ holds algebraically and topologically.

Proof: Only the algebraic identity needs a proof. It is a clearly enough to show that for any $x=\left(x_{k}\right)_{k \in \mathbb{N}} \in L(E)$ there is $n \in \mathbb{N}$ with $x_{k} \in E_{n}$. If this is not satisfied we can find an increasing sequence $(k(n))_{n \in \mathbb{N}}$ in $\mathbb{N}$ such that $x_{k(n)} \notin E_{n}$, for all $n$ in $\mathbb{N}$. We select $\left(\gamma_{k}\right)_{k \in \mathbb{N}} \in \omega \backslash L$ with $\gamma_{k(n)}>0(n \in \mathbb{N})$ and $\gamma_{k}=0$ if $k \notin\{k(n) ; n \in \mathbb{N}\}$. Now since $E_{n}$ is closed, there is $u_{n} \in E^{\prime}$ with $u_{n}\left(x_{k(n)}\right)=\gamma_{k(n)}$ and $u_{n} \mid E_{n}=0$. The equicontinous sequence $\left(u_{n}\right)_{n \in \mathbb{N}}$ defines a continous seminorm as follows:

$$
p(x)=\sup \left\{\left|u_{n}(x)\right| ; n \in \mathbb{N}\right\}
$$

Thus $\left(p\left(x_{k}\right)\right)_{k \in \mathbb{N}} \in L$, a contradiction, since $\gamma_{k} \leq p\left(x_{k}\right)$ for all $k \in \mathbb{N}$.

5. Remark: For an inductive limit $E=$ ind $E_{n}$ and a normal Banach sequence space $(L,\|\|)$, the algebraic coincidence $L(E)=$ ind $L\left(E_{n}\right)$ is a clearly equivalent to $\forall x \in L(E) \exists n \in \mathbb{N}$ with $x \in L\left(E_{n}\right)$. For instance if $(L, \|)=c_{0}$, then $L(E)=$ ind $L\left(E_{n}\right)$ if and only if $E$ is a sequentially retractive (cf [3]). 


\section{References}

1. J. BONET, S. Dierolf, On (LB)-spaces of Moscatelli type, DOGA Tr. J. Math. (to appeat in 1989).

2. J. BONET, S. DIEROLF, Countable inductive limits and the bounded decomposition property, Proc. Roy Ir. Acad. (To appear).

3. K. FLORET, Folgenretraktive Sequenzen Lokalkonvexer Räume, J. reine angew Math 259 (1973), 65-85.

4. G. KöтнE, "Topological vector spaces $I$," Springer, Berlin, Heidelberg, New York, 1979.

5. J. SCHMETS, "Spaces of vector valued continuous functions," Springer, Berlin, Heidelberg, New York, 1983.

J. Bonet: Dept. Maternatica Aplicada

Universidad Politécnica.

C. de Vera

E-46071 Valencia

SPAIN

S. Dierolf: FB IV Mathematik

Postfach 3825

D-5500 Trier

F.R. GERMANY

C. Fernández: Dept. Análisis Matemático

Universidad de Valencia

Dr. Moliner s/n

E-46100 Burjassot

SPAIN

Rebut el 17 de Març de 1989 